\title{
Pathway design effects on synthetic vision head-up displays
}

\author{
Lynda J. Kramer ${ }^{* a}$, Lawrence J. Prinzel III ${ }^{\mathrm{a}}$, Jarvis J. Arthur III ${ }^{\mathrm{a}}$, Randall E. Bailey ${ }^{\mathrm{a}}$ \\ ${ }^{a}$ NASA Langley Research Center, 24 West Taylor Street, Hampton, VA, USA 23681-0001
}

\begin{abstract}
NASA's Synthetic Vision Systems (SVS) project is developing technologies with practical applications that will eliminate low visibility conditions as a causal factor to civil aircraft accidents while replicating the operational benefits of clear day flight operations, regardless of the actual outside visibility condition. A major thrust of the SVS project involves the development/demonstration of affordable, certifiable display configurations that provide intuitive out-thewindow terrain and obstacle information with advanced pathway guidance for transport aircraft. This experiment evaluated the influence of different tunnel and guidance concepts upon pilot situation awareness (SA), mental workload, and flight path tracking performance for Synthetic Vision display concepts using a Head-Up Display (HUD). Two tunnel formats (dynamic, minimal) were evaluated against a baseline condition (no tunnel) during simulated IMC approaches to Reno-Tahoe International airport. Two guidance cues (tadpole, follow-me aircraft) were also evaluated to assess their influence on the tunnel formats. Results indicated that the presence of a tunnel on an SVS HUD had no effect on flight path performance but that it did have significant effects on pilot SA and mental workload. The dynamic tunnel concept with the follow-me aircraft guidance symbol produced the lowest workload and provided the highest SA among the tunnel concepts evaluated.
\end{abstract}

Keywords: Synthetic Vision, Pathway, Tunnel, Head-up Display, Terrain Awareness

\section{INTRODUCTION}

\subsection{Synthetic Vision System}

Limited visibility and reduced situational awareness have been cited as predominant causal factors for Controlled Flight Into Terrain (CFIT) accidents, where a functioning airplane is inadvertently flown into the ground, water, or an obstacle. In commercial aviation, over 30-percent of all fatal accidents worldwide are categorized as CFIT. ${ }^{1}$ Another type of accident typically involving the same causal factors of restricted visibility and compromised situational awareness are runway incursions. Although the number of reported occurrences have decreased for the past two years (339 in FY 2002 and 324 in FY 2003) from an all-time high in FY 2001 (407 occurrences), runway incursions are still a significant threat to aviation safety and operational efficiency. ${ }^{2}$ Finally, the single largest factor causing airport delays is limited runway capacity and increased air traffic separation required when weather conditions fall below visual flight rule operations.

The Synthetic Vision Systems (SVS) project of the National Aeronautics and Space Administration's (NASA) Aviation Safety Program (AvSP) is developing technologies with practical applications that will eliminate low visibility conditions as a causal factor to civil aircraft accidents while replicating the operational benefits of clear day flight operations, regardless of the actual outside visibility condition. To accomplish these safety and situation awareness (SA) enhancements, the SVS concept is designed to provide the pilot an unobstructed view of the world around the aircraft through the display of computer-generated imagery derived from an onboard database of terrain, obstacle, and airport information. To accomplish the operational enhancements, the SVS concept includes the intuitive display of intended flight path by tunnel or pathway-in-the-sky presentations. When coupled with a synthetic or enhanced view of the outside world, the spatially-integrated depiction of the intended aircraft flight path and its relation to the world provides an intuitive, easily interpretable display of flight-critical information for the pilot. Combining this precision guidance capability with SVS terrain information has the potential to eliminate precursors to accidents that have low visibility as a causal factor. The safety outcome of SVS is a display that should help reduce, or even prevent, CFIT,

*lynda.j.kramer@nasa.gov; phone 1757 864-8146 
which is the single greatest contributing factor to fatal worldwide airline and general aviation accidents. ${ }^{1}$ Other safety benefits include reduced runway incursions and loss-of-control accidents. ${ }^{3}$ Operational benefits potentially include more approach and departure options and lower visibility minimums for SVS-equipped aircraft.

Because intuitive perspective displays can naturally depict effective precision pathway (tunnel) guidance concepts, SVS may also significantly improve flight path tracking performance and help meet new FAA required navigation performance (RNP) criteria. RNP is a statement of the navigation performance accuracy necessary for operation within a defined airspace. ${ }^{4}$ RNP type is a designator according to navigational performance accuracy in the horizontal plane (lateral and longitudinal position fixing). This designator invokes all of the navigation performance requirements associated with the applicable RNP number, which is a containment value. For example, RNP-1 means that for at least $95 \%$ of the time the navigational performance in the horizontal plane, or the total horizontal system error, is less than $1.0 \mathrm{nmi}$. In addition to requiring 95\% positioning accuracy for RNP operations, these types of procedures also require integrity of the positioning accuracy at $99.999 \%$ at $2 \times$ RNP number. In our example above with an RNP-1, the position accuracy within $2.0 \mathrm{nmi}$ of the ownship ( $2 \times \mathrm{RNP}$ value of $1.0 \mathrm{nmi}$ ) would have to be guaranteed to be correct $99.999 \%$ of the time to enable RNP-1 operations.

There are three lateral components of navigation error: path definition error, path steering error, and position estimation error. ${ }^{4}$ These errors, defined in the following, represent the total horizontal system error of the airplane and are the difference between the aircraft's true position and desired position:

$>$ The path definition error is the difference between the defined path and the desired path at a specific point.

> The path steering error is the distance from the estimated position to the defined path. It includes both the flight technical error (FTE) and display error. FTE is the accuracy with which the aircraft is controlled as measured by the indicated aircraft position with respect to the indicated command or desired position.

$>$ The position estimation error, also referred to as the ship's actual navigation performance (ANP), is the difference between the true position and the estimated position.

Vertical navigation (VNAV) capability further enhances flight operations by enabling the specification of a flight path vertically for the lateral flight path. VNAV ensures that for at least $99.7 \%$ of the time the navigational performance in the vertical plane, or the total vertical system error, is less than a specified altitude deviation measure based on the airspace being flown in (below 5000 feet MSL, 5000-10000 feet MSL, above 10000 feet MSL) and the type of flight operation (level flight/climb/descent or flight along specified vertical profile) being performed. ${ }^{4}$

There are four vertical components of navigation error: altimetry system error, vertical path steering error, vertical path definition error, and horizontal coupling error. ${ }^{4}$ These errors, defined in the following, represent the total vertical system error of the airplane and are the difference between the aircraft's true vertical position and desired vertical position at the true lateral position:

$>$ Altimetry system error is the error attributable to the aircraft altimetry installation including position effects resulting from normal aircraft flight attitudes.

$>$ The vertical path steering error is the distance from the estimated vertical position to the defined path. It includes both FTE and display error.

$>$ The vertical path definition error is the vertical difference between the defined path and the desired path at the estimated lateral position.

$>$ The horizontal coupling error is the vertical error resulting from horizontal along track position estimation error coupling through the desired path.

\subsection{Related Pathway Research}

Snow and French ${ }^{5}$ examined pilot performance, workload and situation awareness in a Head-Up Display (HUD) SVS study in which HUD-experienced pilots flew complex precision approaches under three visibility conditions (day VMC, night VMC, day IMC), two terrain conditions (with, without), and two flight symbology conditions (pathway-in-thesky, traditional military HUD symbology known as MIL-STD HUD). This study also examined whether the presence of a pathway would lead to cognitive capture and result in reduced SA for required crew duties other than those associated with the pathway. The authors hypothesized that including a pathway with synthetic terrain on a HUD 
results in a conformal symbology set which would naturally draw a pilot's attention to external events. Their hypothesis was tested by placing an aircraft on the approach end of the runway to see if the pilots would recognize the runway incursion. As has been seen in previous NASA studies ${ }^{6,7}$ comparing traditional flight directors to pathway concepts, pilot performance (in terms of flight technical error) was significantly better with the pathway concepts. In addition, the pathway concept decreased workload and enhanced situation awareness. By having increased SA of future-related events, the pilots experienced reduced workload, which allowed for better management of secondary tasks. Results from this study also supported their hypothesis that cognitive capture associated with pathway displays can be alleviated by the application of conformal symbology in a head-up location. The use of conformal pathway symbology in combination with a synthetic runway outline on a HUD facilitated increased SA to events in the far domain that were near to or were overlaid by the symbology.

Researchers at NASA LaRC conducted an experiment examining the efficacy of different tunnel and guidance concepts (see Fig. 1) for head-down synthetic vision displays. ${ }^{9}$ This experiment focused on an SVS head-down primary flight display (PFD) and examined four tunnel concepts (box, minimal, dynamic and pathway) and three guidance symbologies (ball, tadpole, follow-me aircraft). The box tunnel concept (used frequently in past tunnel research) consisted of a series of boxes connected at the corners to form a path within which the pilot is directed to fly. The minimal tunnel concept consisted of a series of "crow's feet" which represented the truncated corners of nominallyconnected 2-dimensional rectangles spaced at $0.2 \mathrm{~nm}$ increments along the desired path. The crow's feet were linearly decreased in brightness so, by $3.0 \mathrm{~nm}$ from own-ship, the brightness of the bottom crow's feet was reduced to zero. The dynamic tunnel concept had the crow's feet grow as a function of path error to provide the pilots feedback on how well they were flying the defined path. The idea of the dynamic tunnel is to minimize clutter when the pilots are flying on path and to alert them as their path error grows by dynamically lengthening the sides of the tunnel in the direction of the path error. The pathway tunnel concept was a variation of the dynamic tunnel concept in which the floor of the tunnel was presented at all times. For both the dynamic and pathway tunnel concepts, when the pilot left the tunnel, the tunnel would change to a trough that resembled three sides of the box tunnel concept. The tunnel was open on one side to "invite" the pilot back into the tunnel.
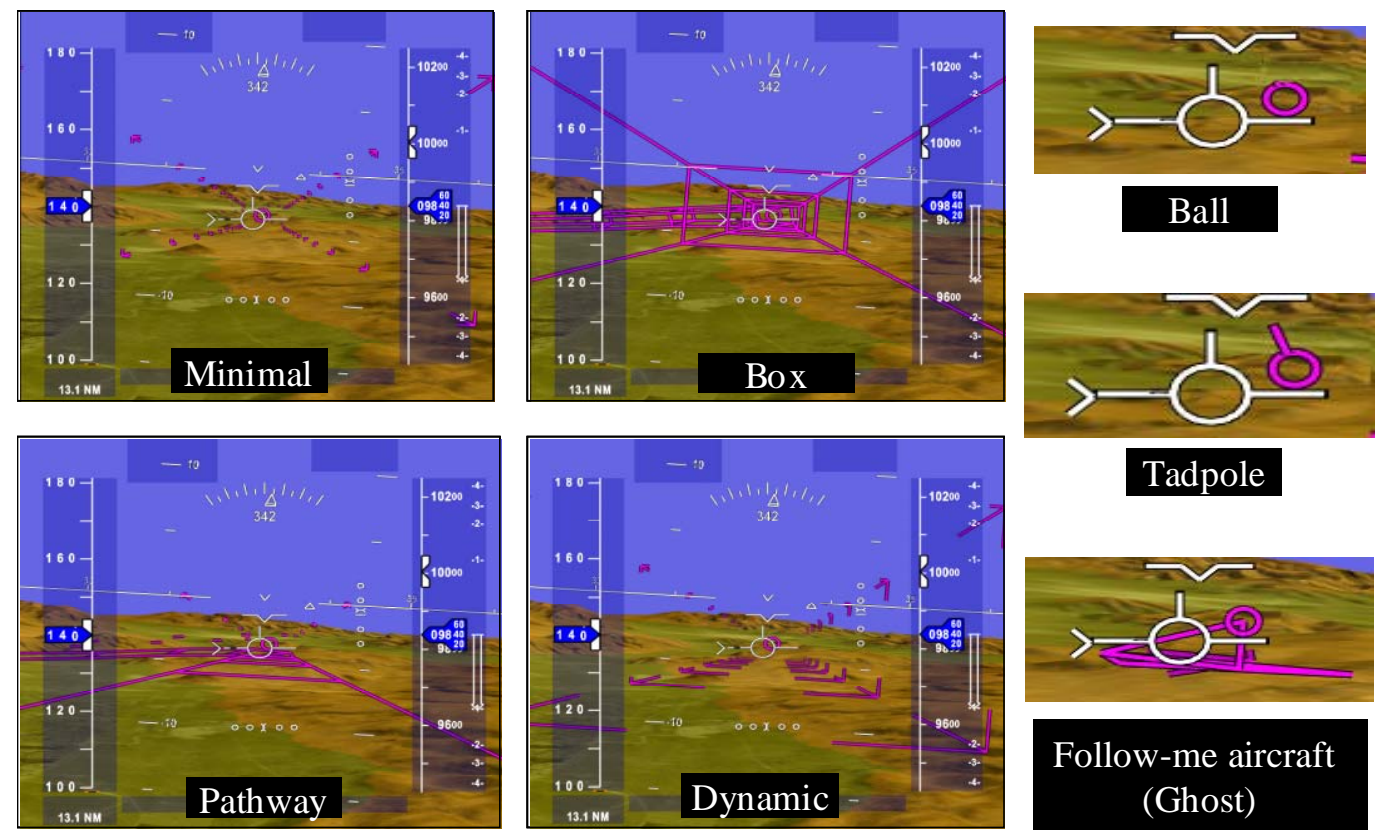

Figure 1. Head-down SVS pathway concepts presented on a Size D display with synthetic terrain of RNO.

All three guidance concepts (ball, tadpole, follow-me aircraft) were driven by the same modified pursuit guidance laws and only differed in their presentation to the pilot. ${ }^{10}$ The guidance symbol was positioned 30 seconds ahead of the ownship on the centerline of the tunnel. Yaw, pitch, and roll attitude changes of the guidance symbol reflected the track and flight path angles of the path at that lead position. The ball symbol was a laterally and vertically integrated 
guidance cue. The tadpole guidance symbol was the same as the integrated ball guidance with added track change information provided by a vertical line. The idea being that the "tadpole" line gives the pilot lateral anticipation of the guidance symbol. The line pointed straight up if the aircraft was flying a perfect lateral track. As the track changed, it rotated left or right in the direction of the track change to denote desired lateral path. The follow-me aircraft (also referred to as the "ghost") guidance symbol provided the pilot with visual yaw, pitch, and roll changes as it flew the path 30 seconds ahead of the ownship. The specific tunnel/guidance concepts tested (see Fig. 1) were: box tunnel/ball guidance, minimal tunnel/ball guidance, pathway tunnel/ball guidance, dynamic tunnel/ball guidance, dynamic tunnel/tadpole guidance, and dynamic tunnel/ghost guidance. The tunnel concepts were evaluated against a baseline concept (no tunnel using the ball guidance symbology). All concepts and the baseline were paired with a navigation display (ND) with a Terrain Awareness and Warning System (TAWS).

Results from this SVS head-down experiment indicated that the presence of a tunnel on an SVS primary flight display had a marginal effect on enhancing lateral flight path performance but significant differences were evident for SA and workload. ${ }^{9}$ For both SA and workload, the no-tunnel condition (baseline) was rated significantly worse (higher workload and lower SA) than any of the tunnel concepts employed. Based on pilot rankings, the dynamic tunnel concept produced the lowest workload and provided the highest SA among the tunnel concepts evaluated. The choice of guidance symbol had no effect on either path performance or workload but did have a significant effect on SA. The ball was rated significantly lower in SA than either the tadpole or ghost but there were no appreciable differences between these latter two guidance symbologies.

\subsection{Current Study}

The present paper reports on an experiment that examined the efficacy of pathway and guidance concepts that may be integrated as part of future synthetic vision head-up displays. Two tunnel formats (dynamic, minimal), which were found to be effective in an SVS head-down display application, were evaluated against a baseline condition (no tunnel) during approaches to Reno-Tahoe International airport (RNO) using the 16R Sparks Visual Arrival under simulated instrument meteorological conditions (IMC). The minimal tunnel was selected as one of the tunnel formats (over the pathway and box tunnel concepts - see Introduction under Related Pathway Research for tunnel definitions) to reduce the scope of the test. The minimal tunnel selection was based on the concern that clutter would be more critical for a HUD than a PFD. In addition, past studies ${ }^{6,7,8}$ have shown that the path deviation indicators (present on all display concepts including baseline in the current study) and the pursuit guidance symbology used in conjunction with the flight path vector have overcome the limited path information provided by the minimal tunnel concept. Two guidance cues (tadpole, ghost) that were found to be equally effective in an SVS head-down application were evaluated to assess their influence on the tunnel format evaluations. A "rare event" runway incursion scenario was also presented to evaluate "attention capture" issues associated with SVS HUDs.

\subsection{Experiment Objectives}

The objectives of this experiment were to: 1) evaluate the efficacy of tunnel/pathway concepts for an SVS head-up display; 2) evaluate different guidance cues and assess their influence on the tunnel format evaluations; 3) compare SVS concepts to a baseline concept in terms of path performance, workload, and situation awareness; and 4) evaluate "attention capture" issues associated with an SVS HUD.

\section{METHODOLOGY}

\subsection{Test Subjects}

Nine pilots, representing four airlines and a major transport aircraft manufacturer, participated in the experiment. All participants were HUD qualified and B-757 type rated. The subjects had an average of 683.7 hours of HUD flying experience and an average of 9.7 years and 12.2 years of commercial and military flying experience, respectively.

\subsection{Simulator}

The experiment was conducted in the Integration Flight Deck (IFD) simulation facility (see Fig. 2) at NASA Langley Research Center (LaRC). The IFD emulates LaRC's Airborne Research Integrated Experimental System (ARIES) Boeing B-757-200 research aircraft's cockpit and provides researchers with a full-mission simulator capability. The cab 
is populated with flight instrumentation, including the overhead subsystem panels, to replicate the B-757 and uses the same pilot controls as ARIES. It also employs a large field of view, out-the-window visual system.

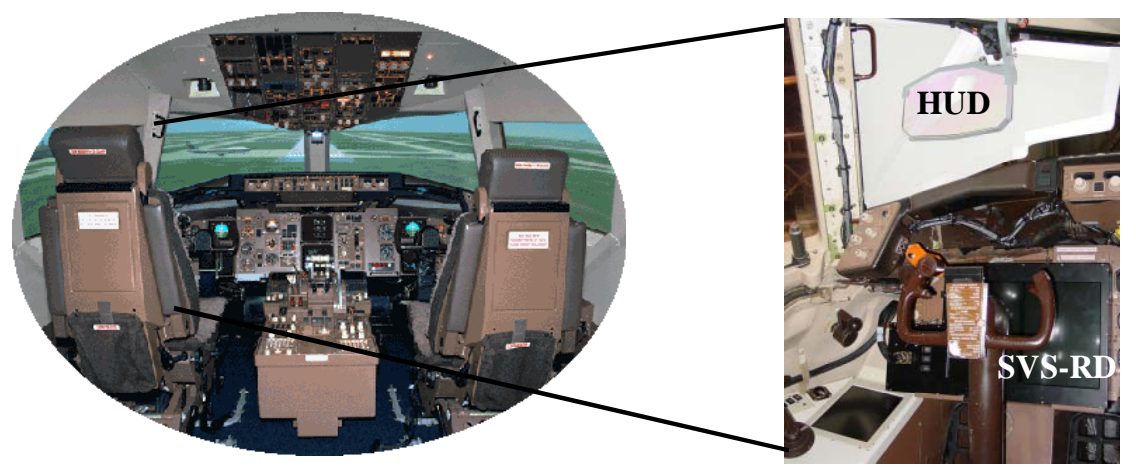

Figure 2. Integration Flight Deck simulation facility with HUD and SVS-RD.

The pilot participants occupied the left seat of the IFD for this experiment. The left seat included the installation of an overhead HUD projection unit and an SVS Research Display (SVS-RD) (see Fig. 2).

\subsubsection{Head-Up Display}

The left-seat, overhead Dassault projection HUD was interfaced with a modified Flight Dynamics Head-Up Guidance System $^{\circledR}$ (HGS)-4000 computer. The HGS-4000 computer is stroke-on-raster capable using an RS-343 raster video format input. The HGS-4000 raster input consisted of the synthetic vision (SV) terrain display. The HGS-4000 "Primary Mode" stroke symbology set was used as the baseline, albeit with the compass rose symbol set and other miscellaneous alphanumeric indicators removed. The modified primary mode stroke symbology set was augmented with the advanced pathway and guidance symbology drawn in stroke as well. The field of view (FOV) of the HUD was measured to be $22^{\circ}$ vertical by $28^{\circ}$ horizontal. Note that to maintain conformality with the outside world, the FOV for the HUD raster image was fixed and could not be varied by the pilot participants. However, brightness and contrast controls were provided: a) Stroke-only brightness; b) Raster image (synthetic terrain imagery) brightness; and c) Raster contrast.

\subsubsection{SVS-RD}

The SVS-RD was an 18.1-inch case containing two high brightness LCD displays, modified for installation over the forward instrument panel cluster on the left hand side of the IFD cockpit (see Fig. 2). The SVS-RD consisted of two separate LCD panels, each with XGA (1024x768) resolution, which rendered a Size D display for the PFD (left display) and ND (right display). The SVS PFD had terrain imagery on it and used the tadpole guidance symbol with no tunnel and the ND had TAWS on it. The SVS-RD covered the normal Boeing 757 captain's displays with the exception of the analog standby instruments (attitude direction indicator, airspeed, and altitude).

\subsection{Tunnel and Guidance Concepts}

Two tunnel concepts (dynamic and minimal) and a baseline (no tunnel) were evaluated (see Fig. 3) on the SVS HUD. Two guidance symbologies (tadpole and ghost) were evaluated with each tunnel concept. The SVS HUD no tunnel baseline condition only used the tadpole guidance symbol. The tadpole symbology is currently used in some military aircraft HUDs (e.g., F-16). 

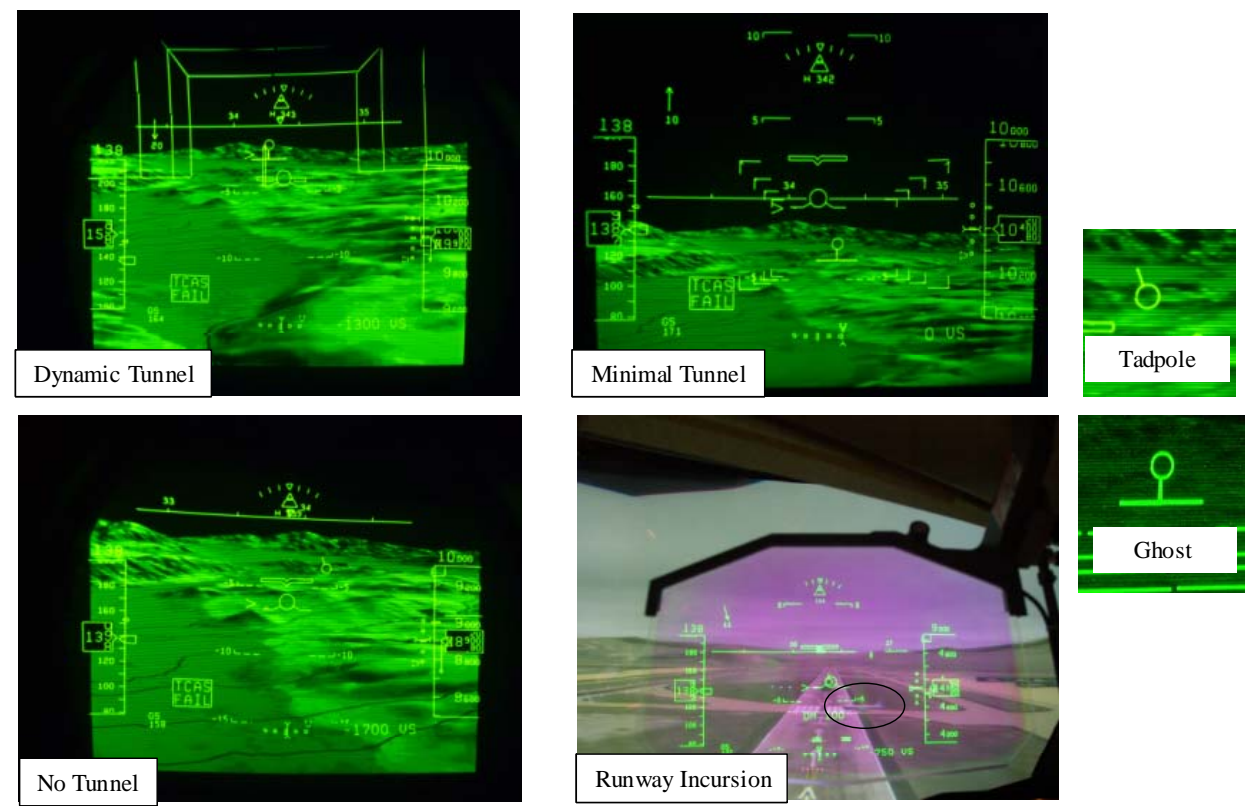

Figure 3. Head-up tunnel and guidance concepts.

\subsection{Evaluation Tasks}

Pilot participants flew the Sparks Visual Arrival to RNO Runway 16R under simulated IMC. The aircraft broke out of the clouds at approximately 500 feet Above Ground Level (AGL). The approach was hand-flown with the autothrottles maintaining an approach speed of 138 knots. The aircraft was configured for landing prior to each run (landing gear down and flaps 30 degrees). Pilot participants were instructed that the run would end at main gear touchdown but that they should perform a go-around if they felt the landing was not safe. In the envisioned SVS HUD concept, a pilot would be able to declutter the tunnel and terrain (separately) whenever it was operationally desirable. However for this experiment, the synthetic terrain and the tunnel were automatically removed (decluttered) by 200 feet AGL. Manual decluttering of the terrain and tunnel was not permitted in this experiment to eliminate decluttering as a possible covariate in the statistical analyses.

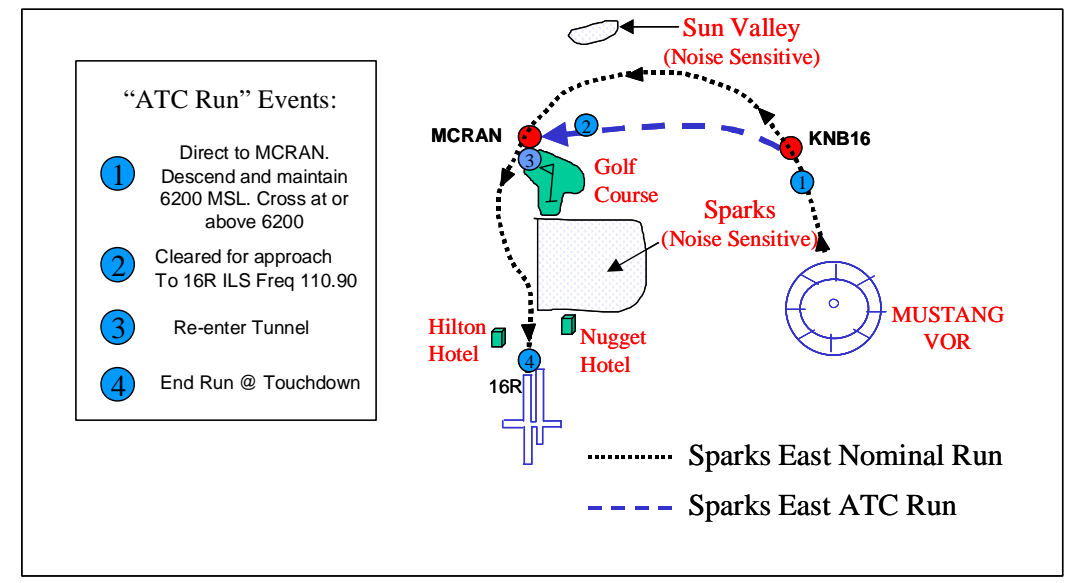

Figure 4. Evaluation tasks for Sparks East Visual Arrival to runway 16R.

Fifteen experimental runs were completed for each pilot. The runs differed from each other on the: 1) initial starting position, outside the tunnel, 2) the pathway/guidance concept, and 3) task scenario. There were three initial starting positions (1000 feet high, 1000 feet right, 500 feet low/500 feet left) that were randomly varied across trials to force the 
pilot to re-enter the tunnel on each run. The five pathway/guidance concepts (no tunnel with tadpole, minimal tunnel with either tadpole or ghost and dynamic tunnel with either tadpole or ghost) were also randomly assigned. Finally, there were two evaluation tasks: 1) nominal Sparks 16R Visual Approach, but flown under simulated IMC, and 2) a "cut the corner" scenario in which the pilot was instructed by a simulated Air Traffic Control call to leave the tunnel and fly "direct to" the MCRAN waypoint (see Fig. 4). The latter scenario required the pilot to utilize the navigation display (i.e., using the turn predictor symbol to acquire the heading) and later, to use the guidance symbology and velocity vector to reenter the tunnel (if present) at the MCRAN waypoint.

A runway incursion (RI) event was randomly assigned to one of the 15 evaluation runs for each pilot. The RI event was used to evaluate "attention capture" for the HUD concept. A B-737 aircraft that had crossed over the hold short line on the approach end of runway 16R created the runway incursion. In the other 14 runs, the B-737 stopped before the holdshort line. The Runway Incursion Prevention System (RIPS) technology ${ }^{11}$ and Traffic Alert and Collision Avoidance System (TCAS) were inhibited during the approaches.

\subsection{Experiment Matrix}

\subsubsection{Measures}

After each run, pilots were administered a run questionnaire consisting of the Air Force Flight Technical Center (AFFTC) Revised Workload Estimation Scale ${ }^{12}$, Situation Awareness Rating Technique (SART) ${ }^{13}$, and six Likert-type (7-point) questions specific to tunnel and guidance symbology evaluation (see Fig. 5). After data collection was completed, pilots were administered two separate Situation Awareness - Subjective Workload Dominance (SASWORD) $)^{14}$ and Subjective Workload Dominance (SWORD) ${ }^{13}$ tests: one for tunnel concept (Baseline, Minimal, Dynamic) comparisons and another for tunnel and guidance cue comparisons. The pilots also participated in a semistructured interview to elicit comments on the tunnel and guidance concepts.

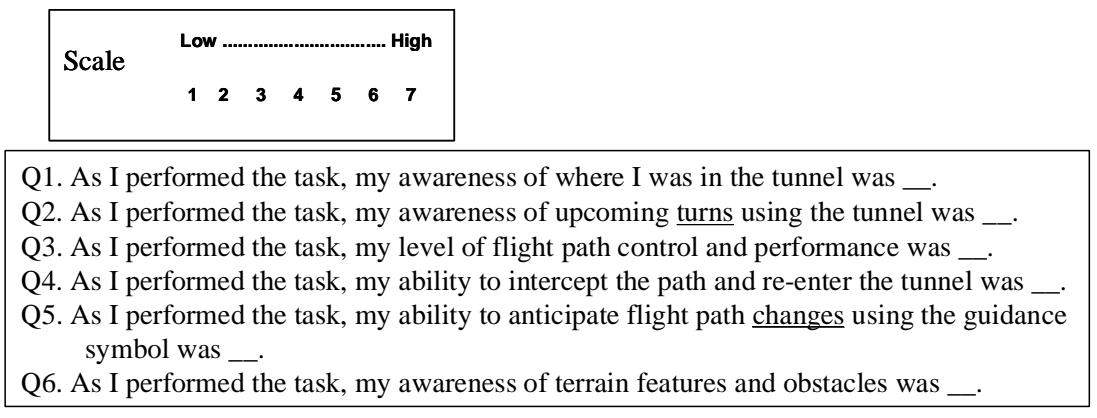

Figure 5. Post-run questions for HUD pathway experiment.

Root-mean-square (RMS) calculations of lateral and vertical path error were used as the measures for flight path performance. In addition, the pilots' FTE was used to determine what level of RNP criteria they could achieve while hand-flying the approach. Simple analysis of variances (ANOVAs) and Student-Newman-Keuls (SNK) post-hoc tests with alpha set at 0.05 were performed.

\subsubsection{Procedure}

The subjects were given a 1-hour briefing to explain the SVS concept and the expected evaluation tasks. After the briefing, a 1-hour training session in the IFD was conducted to familiarize the subjects with the aircraft handling qualities, display symbologies and controls. The 'rare-event' scenario was not discussed, although the pilot's responsibility for maintaining traffic surveillance at all times was stressed. Data collection lasted approximately 3 hours followed by a 1-hour semi structured interview. The entire session including lunch and breaks lasted approximately 6 hours. 


\section{RESULTS}

\subsection{Quantitative Results}

\subsubsection{Path Performance}

Separate ANOVAs were performed on the RMS lateral path error and the RMS vertical path error (see Fig. 6) with tunnel/guidance concept (No tunnel/tadpole, Minimal/tadpole, Minimal/ghost, Dynamic/tadpole, Dynamic/ghost) and pilot as the independent variables. Separate ANOVAs were also performed on the RMS lateral and vertical path error with guidance type (tadpole, ghost) and pilot as the independent variables. No significant differences ( $>$.05) were found for tunnel/guidance type or guidance type for either RMS lateral or RMS vertical path error.

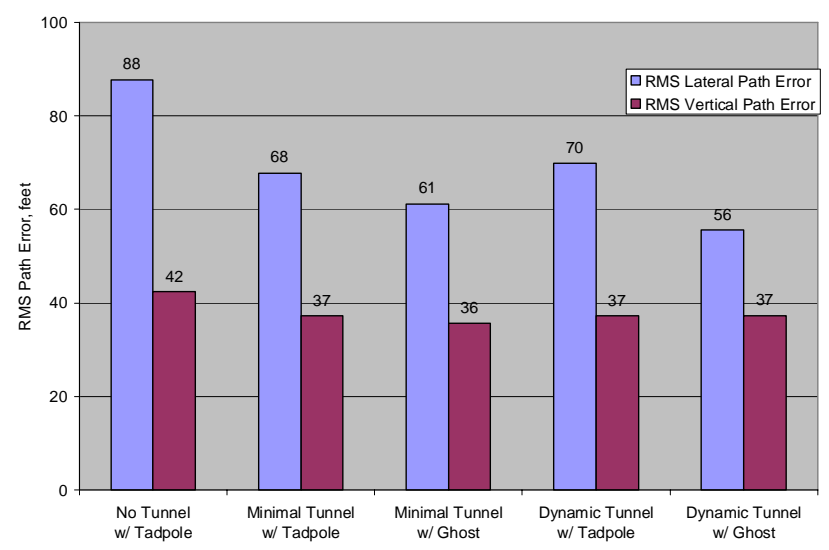

Figure 6. RMS lateral and vertical path error for tunnel/guidance concepts tested.

\subsubsection{RNP Performance}

Lateral Navigation Analyses. Lateral path FTE histograms were generated on the nominal Sparks 16R Approach runs for the five pathway guidance concepts (Baseline with tadpole, Minimal with tadpole or ghost, and Dynamic with tadpole or ghost). Since the initial starting position for each run was outside the tunnel, the histogram analyses were not initiated until the pilot had entered the tunnel for the first time. The path steering error component of the RNP calculation includes both FTE and display error. For this analysis, it was assumed that display error was negligible, so FTE was the only component of path steering error. It was also assumed that the other two components (path definition error and position estimation error) of the RNP calculation would be equivalent across the display concepts evaluated. With these assumptions, all pathway guidance concepts yielded a horizontal FTE navigational accuracy of $0.05 \mathrm{nmi}$ at least $95 \%$ of the time.

Vertical Navigation Analyses. Vertical path FTE histograms were generated on the nominal Sparks 16R Approach runs for the five pathway guidance concepts (Baseline with tadpole, Minimal with tadpole or ghost, and Dynamic with tadpole or ghost). Since the initial starting position for each run was outside the tunnel, the histogram analyses were not initiated until the pilot had entered the tunnel for the first time. The vertical path steering error component of the VNAV performance calculation includes both FTE and display error. For this analysis, it was assumed that display error was negligible so FTE was the only component of vertical path steering error. It was also assumed that the other three components (altimetry system error, vertical path definition error, and horizontal coupling error) of the VNAV performance calculation would be equivalent across the display concepts evaluated. In addition, it was assumed that the pilot was flying a specified vertical profile so that the required vertical navigation performance accuracy was 210 feet. $^{4}$

The SVS HUD concepts with a tunnel yielded a vertical FTE navigational accuracy of 210 feet $100 \%$ of the time; while, the Baseline concept with no tunnel was only able to yield a vertical FTE navigational accuracy of 210 feet $99.4 \%$ of the time. As such, based on the vertical path FTE distributions, the SVS HUD pathway concepts (minimal, dynamic) would enable RNP-type operations along a specified vertical profile of 210 feet and the Baseline concept would not as it 
did not meet the required vertical accuracy $99.7 \%$ of the time. These lateral and vertical FTE navigational accuracies are consistent with previous research results with SVS enhanced displays. ${ }^{6}$

\subsection{Qualitative Results}

\subsubsection{Situation Awareness}

\subsubsection{SA-SWORD Ratings}

Pilots were asked to complete one paired-comparison SA-SWORD ${ }^{14}$ for tunnel/guidance configurations and one for tunnel type. For this exercise, SA was defined as: The pilot's awareness and understanding of all factors that will contribute to the safe flying of their aircraft under normal and non-normal conditions.

Tunnel/guidance concepts. The responses were averaged and the overall rank order from greatest to least SA was: Dynamic tunnel/ghost, Minimal tunnel/ghost, Dynamic tunnel/tadpole, Minimal tunnel/tadpole, and No tunnel/tadpole. An ANOVA was performed on the mean rankings with tunnel/guidance concept and pilot as the independent variables. Tunnel/guidance concept $(\mathrm{F}(4,32)=7.481, \mathrm{p}<.001)$ was highly significant for this measure. Post hoc tests (using Student-Newman-Keuls, SNK, with $\alpha=.05$ ) showed that the Dynamic tunnel/ghost concept had significantly higher SASWORD ratings than all other tunnel/guidance concepts tested (see Fig. 7).

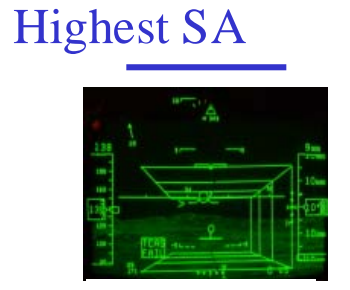

Dynamic/Ghost

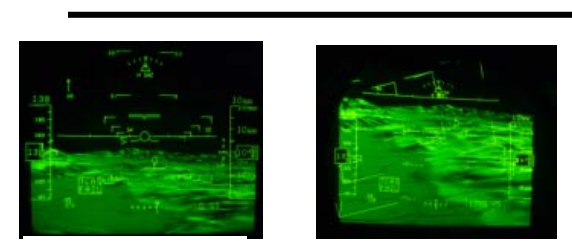

Minimal/Ghost

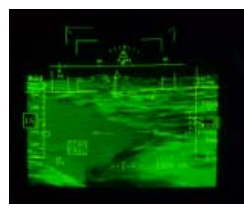

Minimal/Tadpole
Lowest SA

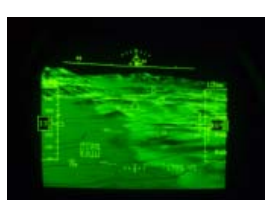

No Tunnel/Tadpole

Figure 7. SA-SWORD rankings.

Tunnel type. The responses were averaged and the overall rank order from greatest to least SA was: Dynamic tunnel, Minimal tunnel, and Baseline (no tunnel). An ANOVA was performed on the mean rankings with tunnel type and pilot as the independent variables. Tunnel type $(\mathrm{F}(2,16)=17.821, \mathrm{p}<.001)$ was highly significant for this measure. Post hoc tests (using SNK with $\alpha=.05$ ) showed 3 unique subsets: 1) Dynamic tunnel, 2) Minimal tunnel, and 3) No tunnel. The Dynamic tunnel provided significantly higher SA compared to the other two tunnel types (minimal and baseline) and the Minimal tunnel provided significantly higher SA than the Baseline concept with no tunnel.

\subsubsection{SART Ratings}

After each run, pilots were asked to complete the SART for the tunnel/guidance concept they had just flown. The overall rank order of the mean ratings from greatest to least SA was: Dynamic tunnel/ghost, Minimal tunnel/ghost, Minimal tunnel/tadpole, Dynamic tunnel/tadpole, and No tunnel/tadpole. An ANOVA was performed on the mean rankings with tunnel/guidance concept and pilot as the independent variables. Tunnel/guidance concept $(\mathrm{F}(4,122)=3.701, \mathrm{p}=.007)$ was highly significant for this measure. Post hoc tests (using SNK with $\alpha=.05$ ) showed two overlapping subsets: 1) Dynamic tunnel/ghost, Minimal tunnel/ghost, Minimal tunnel/tadpole, Dynamic tunnel/tadpole and 2) Dynamic tunnel/tadpole, No tunnel/tadpole (Baseline condition).

\subsubsection{Mental Workload}

\subsubsection{SWORD Ratings}

Pilots were asked to complete one paired-comparison SWORD ${ }^{13}$ for tunnel/guidance configurations and one for tunnel type. The SWORD for this experiment was designed to allow a statistical analysis of the pilot's subjective assessment 
of mental workload. For this exercise, Mental Workload was defined as: The amount of cognitive resources available to perform a task and the difficulty of that task.

Tunnel/guidance concepts. The responses were averaged and the overall rank order from least to greatest workload was: Dynamic tunnel/ghost, Minimal tunnel/ghost, Dynamic tunnel/tadpole, Minimal tunnel/tadpole, and No tunnel/tadpole. An ANOVA was performed on the mean rankings with tunnel/guidance concept and pilot as the independent variables. Tunnel/guidance concept $(\mathrm{F}(4,32)=30.456, \mathrm{p}<.001)$ was highly significant for this measure. Post hoc tests (SNK with $\alpha=.05$ ) showed that the Baseline concept had significantly higher SWORD ratings than all the other tunnel/guidance concepts tested. There were no appreciable differences between the four concepts that employed a tunnel.

Tunnel type. The responses were averaged and the overall rank order from least to greatest workload was: Minimal tunnel, Dynamic tunnel, and Baseline (no tunnel). An ANOVA was performed on the mean rankings with tunnel type and pilot as the independent variables. Tunnel type $(\mathrm{F}(2,16)=24.599, \mathrm{p}<.001)$ was highly significant for this measure. Post hoc tests (using SNK with $\alpha=.05$ ) showed that the Baseline concept was rated as having significantly more workload than either the Dynamic or Minimal tunnel formats. There were no appreciable differences between the Minimal and Dynamic tunnel types.

\subsubsection{AFFTC Workload Estimate Ratings}

After each run, pilots were asked to provide a workload rating on the tunnel/guidance concept they had just flown by using the AFFTC Workload Estimate Scale. The overall rank order of the mean ratings was Minimal tunnel/ghost, Dynamic tunnel/ghost, Minimal tunnel/tadpole, Dynamic tunnel/tadpole, and No tunnel/tadpole. An ANOVA was performed on the mean rankings with tunnel/guidance concept and pilot as the independent variables. No significant differences ( $p>.05)$ were found for this workload rating among the tunnel/guidance concepts. Overall, the pilots rated the Baseline concept (3.0/7.0) to be slightly higher in workload than the Minimal tunnel (2.8/7.0) or Dynamic Tunnel (2.9/7.0). A rating of "3.0" reflects "moderate activity" that is "easily managed".

\subsubsection{Post-run Questionnaire Results}

Six post-run questions (see Fig. 5) were asked of each pilot to help assess specific subjects of interest while flying the approaches with the tunnel/guidance concepts. An ANOVA was performed on the mean rating (1 = "high"; $7=$ "low") for each of those post-run questions with tunnel/guidance type (five levels: Baseline/Tadpole; Minimal/Tadpole; Minimal/Ghost; Dynamic/Tadpole; Dynamic/Ghost) and pilot as the independent variables. Only Questions 2 and 5 of the six post-run questions (Fig. 5) had significant differences among the tunnel/guidance concepts evaluated.

Post-run question \#2 was asked of each subject pilot to help assess his awareness of upcoming turns when flying the different tunnel concepts. As this query focused specifically on the tunnel, the Baseline (no tunnel) concept was excluded from this analysis. Tunnel/guidance type $(\mathrm{F}(3,95)=2.774, \mathrm{p}=.047)$ was significant for this rating. Post hoc tests (using SNK with $\alpha=.05$ ) showed that the pilots were least aware of upcoming turns with the minimal tunnel/tadpole guidance symbol concept. Two overlapping subsets were found: 1) Minimal/tadpole (6.0/7.0), Dynamic/tadpole (6.2/7.0), Minimal/ghost (6.2/7.0) and 2) Dynamic/tadpole, Minimal/ghost, Dynamic/ghost (6.4/7.0). The pilots rated the Dynamic tunnel/ghost guidance symbol concept as providing them significantly more awareness of upcoming turns compared to the Minimal tunnel/tadpole guidance symbol concept.

Post-run question \#5 was asked of each subject pilot to help assess his ability to anticipate flight path changes using the guidance symbology when flying with the different tunnel/guidance concepts. Tunnel/guidance type $(\mathrm{F}(4,122)=8.479$, $\mathrm{p}<.001$ ) was highly significant for this rating. Post hoc tests (using SNK with $\alpha=.05$ ) showed two unique subsets: 1) No tunnel/tadpole (5.2/7.0) and 2) Minimal/ghost (5.9/7.0), Minimal/tadpole (5.9/7.0), Dynamic/tadpole (6.0/7.0), Dynamic/ghost (6.1/7.0). These subsets appear to indicate that the presence of the tunnel, rather than the guidance symbology, affected the pilot's ability to anticipate flight path changes.

\subsubsection{Semi-structured Interview Results}

A semi-structured interview was conducted after the final experimental run. A number of Likert (1 to 7) questions were administered to evaluate 1) the effectiveness of the different tunnel (minimal, dynamic) and guidance (tadpole and 
ghost) concepts and 2) the workload associated with leaving the tunnel and reentering the tunnel at the MCRAN waypoint. Several interesting results and trends were found. For example, although there was no significant difference in rating for effectiveness of tunnels for straight or curved tunnel segments, the trend was that the Dynamic tunnel concept (6.6/7.0 for straight segments \& 6.1/7.0 on curved segments) was always rated more effective than the minimal tunnel concept (6.0/7.0 for straight segments \& 5.8/7.0 on curved segments). These effectiveness ratings of 6 or greater for both tunnel concepts indicated that both were very effective for depicting flight path for both straight and curved tunnel segments. An ANOVA was performed on pilot responses to the effectiveness of guidance symbology (tadpole, ghost) for situation awareness of future flight path for both straight and curved path segments. A highly significant result was found only for the curved/banked flight path segments, $F(1,8)=19.360, p=.002$. Pilots rated the tadpole (5.0/7.0) significantly worse than the ghost (6.2/7.0) for SA of future flight path on curved tunnel segments.

Another interesting finding was that pilots rated the Baseline concept (4.1) to have significantly more workload to intercept the path during the "cut-the-corner" scenario, $\mathrm{F}(4,32)=8.995, \mathrm{p}<.001$, compared to the four tunnel concepts. The Dynamic/ghost concept (2.2) was also rated as having significantly less workload than the Minimal/tadpole (4.1) concept, but it had no appreciable differences with the Minimal/ghost (2.6) or Dynamic/tadpole (2.7) concepts. A workload rating of 2 indicates low workload while a workload rating of 4 indicates moderate workload.

\subsubsection{Runway Incursion Detection}

Only one (1/9) of the pilots failed to notice the B-737 aircraft that had crossed over the hold short line onto the active runway. During the post-experimental interview, this pilot acknowledged seeing the aircraft but felt it was too late to initiate the go-around and hence decided to land. The pilot felt that the situation did not pose any danger since he could land the aircraft further down the runway well beyond the incursion aircraft. As discussed earlier, at 200 feet AGL the tunnel and the raster terrain were automatically removed (decluttered) from the HUD, while the guidance cue and other flight symbology were still present on the HUD. These results support that the NASA SVS HUD concept does not significantly decrease unexpected event detection. However, to further safeguard against incursions, the NASA SVS HUD concept will incorporate runway incursion prevention system (RIPS) technology. ${ }^{11}$

\section{CONCLUSIONS}

Two tunnel formats (dynamic, minimal), which were found to be effective in a Synthetic Vision Systems (SVS) headdown display application, were evaluated against a baseline condition (no tunnel) during approaches to Reno-Tahoe International airport (RNO) using the 16R Sparks Visual Arrival under simulated instrument meteorological conditions (IMC). These tunnel formats were chosen for the present experiment as they represented the best candidates for SVS head-up display (HUD) applications due to clutter concerns. In addition, two guidance cues (tadpole, follow-me aircraft) that were found to be equally effective in an SVS head-down application were evaluated to assess their influence on the tunnel format evaluations. Pilots reported greater situation awareness (SA) and reduced pilot workload, and had lower flight technical error (FTE) while operating with the SVS HUD tunnel/guidance concepts compared to the Baseline concept without a tunnel. The dynamic tunnel concept with the follow-me aircraft guidance symbol produced the lowest workload and provided the highest SA among the tunnel/guidance concepts evaluated. In addition, the follow-me aircraft symbol was rated as being the most effective guidance cue for SA of future flight path for both straight and curved path segments. These results confirmed findings from an SVS head-down experiment that also found the dynamic tunnel and follow-me aircraft guidance concept to be the best candidate for SVS primary flight display applications.

It was hypothesized that the increased path precision provided by the SVS pathway and pursuit guidance presentation would enable pilots to make manual approaches within required navigation performance (RNP) accuracies that normally require RNAV capabilities. The lateral navigation analyses confirmed that FTE for all the SVS display concepts (including baseline) achieved an accuracy of $0.05 \mathrm{nmi}$ for at least $95 \%$ of the approach. The presence of a tunnel did not reduce a pilot's lateral FTE but it did affect vertical path performance. All tunnel concepts achieved the required vertical accuracy of 210 feet $100 \%$ of the time. The baseline concept (no tunnel) was flown to the required vertical accuracy $99.4 \%$ of the time, which is slightly outside the RNP permissible limits of $99.7 \%$.

Based on these results, research is currently being conducted at NASA Langley to enhance the dynamic tunnel concept with tactical and strategic display information to help realize 4D RNP capability. A flight test evaluation is anticipated 
in 2004 by the NASA/Langley Research Center under NASA's Aviation Safety, Synthetic Vision System Project to examine a synthetic vision system that integrates the enabling technologies (Runway Incursion Prevention System, Synthetic Vision Sensors, and Database Integrity Monitoring Equipment) of SVS. The research will focus on the integration of runway incursion prevention technologies, surface map displays, integrity monitoring, enhanced vision sensors, SVS navigation displays, and enhanced synthetic vision primary flight and HUD displays. Together, such a synthetic vision system may considerably help meet national aeronautic goals to "reduce the fatal accident rate by a factor of 5" and to "double the capacity of the aviation system", both with 10 years. ${ }^{15}$

\section{REFERENCES}

1. Boeing (1998). Statistical summary of commercial jet aircraft accidents, Worldwide Operations, 1959-1997. Seattle, WA: Airplane Safety Engineering, Boeing Commercial Airplane Group.

2. FAA (2003). FAA Runway Safety Report - Runway Incursion Trends at Towered Airports. FAA Office of Runway Safety, July 2003.

3. Williams, D., Waller, M., Koelling, J., Burdette, D., Doyle, T., Capron, W., Barry, J., \& Gifford, R. (2001). Concept of operations for commercial and business aircraft synthetic vision systems. NASA Langley Research Center: NASA Technical Memorandum TM-2001-211058.

4. RTCA (2000). Minimum Aviation System Performance Standards: Required Navigation Performance for Area Navigation (RTCA DO-236A). Washington, D.C.: RTCA, Incorporated.

5. Snow, Michael P. \& French, Guy A. (2002). Effects of primary flight display symbology on workload and situation awareness in a head-up synthetic vision display. Air Force Research Laboratory, 29 August.

6. Kramer, L.J., Prinzel, L.J., Bailey, R.E., \& Arthur, J.J. (2003). Synthetic vision enhances situation awareness and RNP capabilities for terrain-challenged approaches. Proceedings of the American Institute of Aeronautics and Astronautics Third Aviation Technology, Integration, and Operations Technical Forum, AIAA 2003-6814, $1-11$.

7. Glaab, L.J., Kramer, L.J., Arthur, T., \& Barry, J.S. (2003). Flight test comparison of synthetic vision display concepts at Dallas/Fort Worth International airport. NASA Langley Research Center: NASA Technical Paper TP-2003-212177.

8. Arthur, J.J., Prinzel, L.J., Kramer, L.J., Bailey, R.E., \& Parrish, R.V. (2003). CFIT Prevention Using Synthetic Vision. SPIE. In Proceedings of SPIE, Enhanced and Synthetic Vision 2003, Editor: Jacques G. Verly, Volume 5018 paper 16 Apr.

9. Prinzel, L.J., Arthur, J.J., Kramer, L.J., \& Bailey, R.E. (in press). Pathway concepts experiment for head-down synthetic vision displays. SPIE. In Proceedings of SPIE, Enhanced and Synthetic Vision 2004, Editor: Jacques G. Verly, Volume 5424 paper 12 Apr.

10. Merrick, V.K. \& Jeske, J.A. (1995). Flightpath synthesis and HUD scaling for V/STOL terminal area operations. NASA Langley Research Center: NASA Technical Memorandum TM-1995-110348.

11. Jones D.R., Quach, S.D., \& Young, S.D. (October 14, 2001). Runway Incursion Prevention System Demonstration and testing at Dallas/Fort Worth International Airport. $20^{\text {th }}$ Annual Digital Avionics Systems Conference (DASC). Daytona Beach, FL.

12. Ames, Lawrence L. \& George, Edward J. (1993). Revision and verification of a seven-point workload estimation scale. Air Force Flight Technical Center: AFFTC-TIM-93-01.

13. AIAA (1993). Guide to Human Performance Measurement. Washington D.C.: American Institute of Aeronautics and Astronautics.

14. Vidulich, M.A. \& Hughes, E.R. (1991). Testing a subjective metric of situation awareness. Proceedings of the Human Factors \& Ergonomics Society, 35, 1307-1311.

15. National Aeronautics and Space Administration (2001). Aerospace Technology Enterprise. Washington, D.C.: NASA. 